\title{
Instrumentos de avaliação da qualidade de vida na demência
}

Ana Paula Pinheiro*

\section{RESUMO}

Objetivos: Este estudo teve como objetivo conhecer os instrumentos específicos para avaliação da qualidade de vida (QoL) dos doentes com demência. Compara as diferentes medidas em relação a diversos parâmetros, nomeadamente população-alvo quanto ao grau de demência, dados psicométricos e principais características.

Fontes de dados: MEDLINE/PubMed e RCAAP.

Métodos de revisão: Pesquisa de artigos publicados entre 1 de janeiro de 1986 e 31 de dezembro de 2016 com os termos MeSH Quality of Life, Scales, Measures, Dementia e Alzheimer Disease.

Resultados: Obtiveram-se 67 trabalhos, dos quais foram selecionados 18. Nestes foram encontrados 10 questionários de avaliação da QoL em indivíduos com demência.

Conclusões: Dos instrumentos específicos encontrados, apenas um foi traduzido, adaptado e validado para a cultura portuguesa.

Palavras-chave: Qualidade de vida; Escalas; Instrumentos; Demência; Doença de Alzheimer.

\section{INTRODUÇÃO}

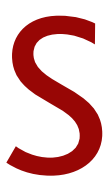
egundo a Organização Mundial da Saúde (OMS), a demência é uma síndroma, geralmente de natureza crónica ou progressiva, caracterizada pela deterioração da função cognitiva para além do que se poderia considerar consequência de um envelhecimento normal. A demência afeta a memória, o pensamento, a orientação, a compreensão, o cálculo, a capacidade de aprendizagem e a linguagem. A deterioração cognitiva pode acompanhar-se ou ser precedida por deterioração do controlo emocional, comportamento social ou motivação. A doença de Alzheimer é a causa mais comum de demência. A investigação dos défices cognitivos é normalmente realizada com recurso ao Mini Mental State Examination (MMSE).

O grupo Alzheimer Europe conduziu um projeto financiado pela Comissão Europeia - European Collaboration on Dementia (Eurocode) -, que calculou o número de europeus com demência em 9,2 milhões. Face ao envelhecimento da população nos Estados-membros da União Europeia, os especialistas preveem uma duplicação destes valores em 2040 na Europa Ocidental, podendo atingir o triplo na Europa de Leste. Todos

* Médica Assistente Graduada de Medicina Geral e Familiar. Coordenadora. USF Viseu-Cidade. Diretora do Núcleo de Internato de Medicina Geral e Familiar de Viseu. os anos, 1,4 milhões de cidadãos europeus desenvolvem demência, o que significa que a cada 24 segundos um novo caso é diagnosticado. ${ }^{1}$

Em Portugal estima-se que existam cerca de 153.000 pessoas com demência, 90.000 com doença de Alzheimer.

A OMS assume a qualidade de vida (QoL) como a "percepção do indivíduo acerca da sua posição na vida, no contexto cultural e sistema de valores nos quais está inserido e em relação aos seus objectivos, expectativas, padrões e preocupações". ${ }^{2}$

A qualidade de vida relacionada com a saúde é entendida, desde a segunda metade do século passado, como uma parte da QoL geral do indivíduo que se relaciona direta ou indiretamente com a saúde, a doença e suas repercursões. ${ }^{3}$

O conceito de QoL relacionado com a saúde é multidimensional, relacionando-se com a dimensão biopsicossocial dos indivíduos. A dimensão biológica/funcional representa os sinais e sintomas físicos relacionados com a doença ou tratamento e a capacidade de autonomia funcional do indivíduo (incluindo o autocuidado, a mobilidade e a capacidade de desempenho do papel social). A dimensão psicológica engloba a perceção global de saúde, o estado mental e o emocional. A componente social inclui a interação dos doentes 
com a sua família e o meio social mais alargado, assim como a sua participação em atividades sociais. A QoLé uma avaliação global, um somatório de todos os componentes. ${ }^{4}$

Dadas as perdas cognitivas que ocorrem no paciente com demência e a subjetividade dos relatos, a avaliação da QoL nestes casos torna-se um desafio. No entanto, nas fases iniciais da demência os pacientes conseguem expressar-se sobre a sua QoL, participando ativamente no preenchimento dos questionários de avaliação. ${ }^{5-6} \mathrm{~A}$ perceção sobre a QoL não está relacionada com o grau de défice cognitivo nos estádios ligeiros a

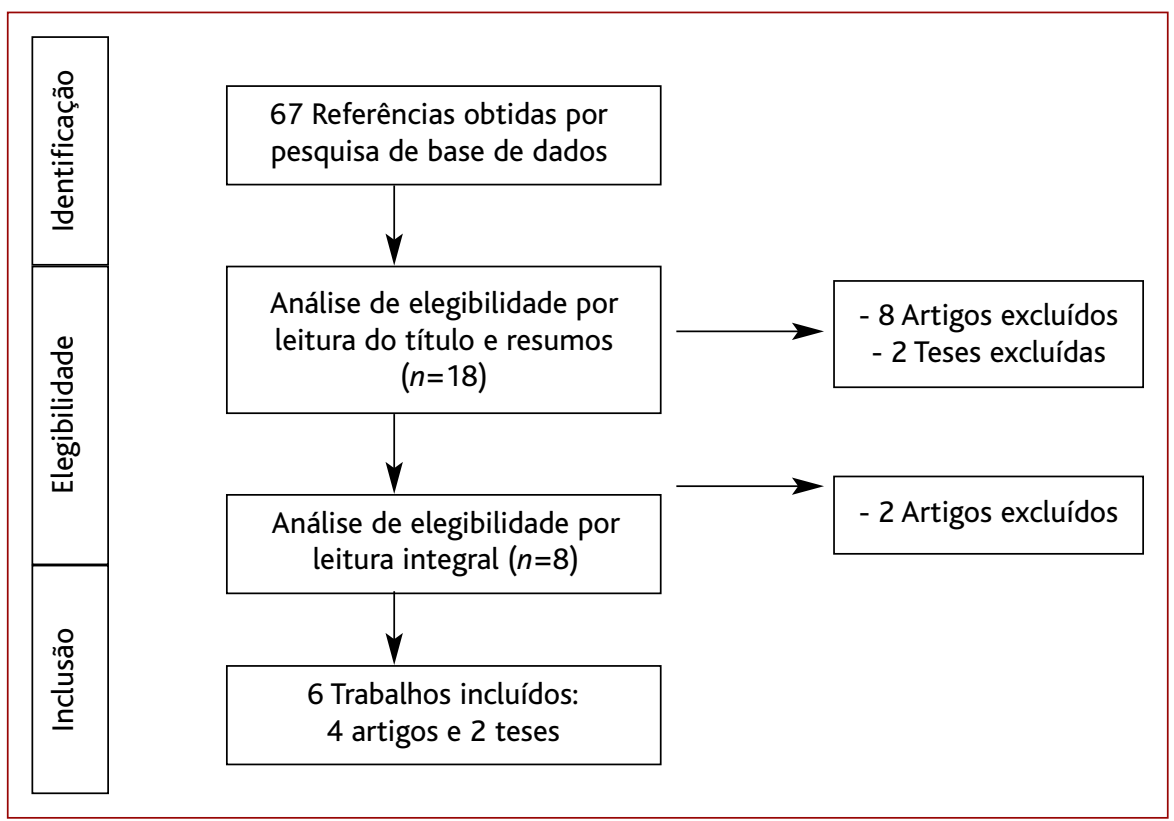

Figura 1. Fluxograma de seleção dos trabalhos. moderados de demência. ${ }^{7-8}$

Inúmeras escalas de avaliação de QoL na demência tiveram como base conceptual o modelo teórico de QoL para idosos, desenvolvido por Lawton. ${ }^{9}$ As definições de QoL na demência baseiam-se em aspetos gerais da QoL, incluindo a saúde física e mental, competências cognitivas, relacionamento familiar e social, atividade profissional, atividades de lazer e sucesso económico. ${ }^{10}$

Com o aparecimento de novos tratamentos para a demência, com a melhoria do funcionamento cognitivo, muitos investigadores e clínicos referem que é importante avaliar se também existem benefícios na QoL do doente. ${ }^{711}$ A avaliação da QoL poderia funcionar como uma medida da satisfação do doente e dos seus cuidadores com os tratamentos instituídos. No entanto, a QoL reportada pelas pessoas com demência não é coincidente com a reportada pelos seus cuidadores, que tendem a atribuir pontuações mais baixas do que os próprios., ${ }^{72-13}$

O presente trabalho teve como objetivo pesquisar na literatura as evidências de validade dos questionários elaborados para avaliar a QoL de doentes com demência.

\section{MÉTODOS}

Foi realizada uma pesquisa nas bases de dados MEDLINE/PubMed e RCAAP, utilizando os termos MeSH Quality of Life, Scales, Measures, Dementia e Alzheimer
Disease. Incluíram-se os artigos publicados entre 1 de janeiro de 1986 e 1 de dezembro de 2016.

Foram incluídos trabalhos sobre instrumentos de avaliação da qualidade, com estudos de validade, em indivíduos com demência. Foram excluídos todos os trabalhos que tratavam de instrumentos sem estudos de validade ou que não mencionavam instrumentos de avaliação da qualidade em demência.

As referências bibliográficas encontradas nos artigos selecionados também foram incluídas.

\section{RESULTADOS}

Obteve-se um total de 67 (28 PubMed + 39 RCAAP) trabalhos, dos quais foram selecionados $18(14+4)$, que incidiam especificamente sobre os questionários de avaliação da QoL na demência. O fluxograma de seleção dos trabalhos está representado na Figura 1.

Foram encontrados 10 questionários de avaliação da QoL em indivíduos com demência, que se descrevem.

\section{Questionários internacionais de avaliação da Qualidade de Vida na Demência}

\section{a) Dementia Quality of Life Instrument (DQoL) ${ }^{14}$}

Foi a primeira escala a ser construída, de acordo com as recomendações da FDA, a partir da revisão biblio- 
gráfica e consulta com três grupos: pacientes com demência ligeira a moderada (MMSE $=12-21$ ), cuidadores e profissionais de saúde. A informação recolhida permitiu identificar uma referência conceptual de QoL, incluindo áreas como a capacidade física, mobilidade, atividades da vida diária, interação social, interação com o ambiente, capacidade para se divertir e perceção geral da saúde.

É uma escala de autorresposta pelos pacientes com demência em fases iniciais, composta por 29 perguntas específicas e uma geral ("No todo, como avaliaria sua qualidade de vida?”). As perguntas específicas estão agrupadas em cinco áreas: estados emocionais positivos (seis itens), estados emocionais negativos (onze itens), relações sociais (três itens), autoestima (quatro itens) e sentido estético (cinco itens). As respostas são dadas pelo paciente através de uma escala visual com cinco opções de resposta (nunca, algumas vezes, ocasionalmente, habitualmente e quase sempre), com um tempo médio de aplicação de cerca de dez minutos. Quanto mais alta a pontuação, melhor a QoL.

A coerência interna desta medida foi considerada moderada a alta (alfa de Cronbach $=0,67$ a 0,89; média $=0,80$ ). Tem boa fiabilidade, não tendo sido encontrada diferença significativa entre os pacientes com demência ligeira e demência moderada.

\section{b) Activity and Affect Indicators of Quality of Life ${ }^{15}$}

Este instrumento de avaliação apenas avalia duas dimensões - as atividades realizadas e o estado emocional. Pode ser usado nas várias fases da demência, uma vez as questões devem ser respondidas pelo cuidador. Quinze questões referem-se às atividades realizadas dentro e fora de casa, sendo classificadas tendo em conta a sua frequência no período de uma semana. A avaliação do estado emocional (prazer, raiva, ansiedade, depressão, interesse e satisfação) baseia-se nas expressões faciais e corporais que ocorrem no decorrer das duas últimas semanas, numa escala que varia numa frequência de nunca a igual ou superior a três vezes por dia (um a cinco pontos).

Os estudos preliminares de fiabilidade e validade da escala foram realizados por entrevista a 130 cuidadores de pacientes com doença de Alzheimer, com um alfa de Cronbach para as questões de estado emocional de 0,77 e acima de 0,60 para doze das quinze questões relacio- nadas com as atividades realizadas. A validade da escala foi provada por correlação entre a frequência das atividades realizadas e as pontuações obtidas pelo MMSE. As medidas dos estados emocionais mostraram associações variáveis com a gravidade da demência.

\section{c) Quality of Life-Alzheimer's Disease (QOL-AD) ${ }^{7,11}$}

É uma escala genérica de avaliação da QoL na demência desenhada para pacientes com doença de Alzheimer que vivem na comunidade. Foram selecionadas perguntas que refletem as áreas de QoL do modelo de Lawton, com validação semântica por pacientes com doença de Alzheimer, cuidadores e peritos em geriatria.

Aborda treze parâmetros distintos relacionados com a saúde física, disposição, humor, memória, família, relações interpessoais, capacidade de executar tarefas, capacidade de participar em atividades de lazer, situação económica e vida em geral.

As questões referem-se ao momento presente, com quatro opções de resposta: mau (1), satisfatório (2), bom (3) e excelente (4). A escala pode ser preenchida pelo próprio paciente, pelo cuidador ou por ambos em separado. A pontuação dos pacientes tem uma ponderação de 2 e é adicionada à pontuação do cuidador, o total é dividido por 3. Tem um tempo médio de preenchimento de 10 minutos ou menos, quando preenchido pelo cuidador. A pontuação total varia entre 13 e 52 pontos; quanto maior o valor obtido, melhor a QoL.

As propriedades psicométricas foram inicialmente avaliadas em 1999 com uma amostra de 77 pacientes com doença de Alzheimer e seus cuidadores e, posteriormente, em 2002 com uma amostra de 177 doentes. A coerência interna (alfa do Cronbach) variou de 0,84 a 0,88 para pacientes e cuidadores. A validade foi testada por correlações entre pontuações da QoL-AD e do Mini-Mental, relatórios de atividades instrumentais de vida diária, depressão e participação em eventos agradáveis.

Sintomas depressivos ou burnout dos cuidadores influenciam fortemente os relatos dos cuidadores, condicionando resultados de QoL sistematicamente inferiores aos atribuídos pelo paciente.

\section{d) The Quality of Life in Late-Stage Dementia Scale (QUALID) $)^{16}$}

É uma escala elaborada a partir de uma seleção de questões de um outro instrumento de avaliação - Acti- 
vity and Affect Indicators of Quality of Life - para ser aplicada em pacientes institucionalizados com demência num estádio avançado. É preenchida pelo cuidador num tempo médio de cinco minutos. Avalia as atividades diárias através de onze comportamentos e estados emocionais observáveis nos sete dias anteriores.

Foi avaliada em 42 doentes institucionalizados, sendo exigido um contacto mínimo de 30 horas semanais aos cuidadores para que pudessem responder. A coerência interna foi boa, com um alfa de Cronbach de 0,77.

Não foram encontradas correlações entre as pontuações totais do QUALID e as pontuações do MMSE, mas foram encontradas correlações moderadas com as pontuações da Escala de Depressão Geriátrica $(r=0,36)$.

\section{e) Quality of Life Assessment Schedule in Dementia (QOLAS) ${ }^{17}$}

É uma escala de qualidade de vida genérica adaptada para doentes com demência ligeira a moderada, aplicadas aos próprios pacientes. Avalia várias dimensões, nomeadamente a integridade física, psicológica, social e familiar, as atividades habituais e as funções cognitivas. Os pacientes avaliam o quanto um problema tem impacto na sua vida, numa escala de seis pontos (sendo 0 $=$ nenhum problema e $5=$ não poderia ser pior) em cada uma das questões, variando a pontuação final de 0 a 50 . Quanto maior o valor obtido, pior a Qualidade de Vida.

Foi testada num grupo de 22 pacientes com demência ligeira a moderada e seus cuidadores, com uma coerência interna de 0,78 (alfa de Cronbach). Quanto à validade semântica, os pacientes foram capazes de entender as questões da escala e de descrever (quantitativa e qualitativamente) a sua QoL, pelo que os autores admitiram que estes podem avaliar sozinhos a própria QoL. A validade de constructo foi indicada por uma perceção de QoL mais elevada para pacientes com menores compromissos para o desempenho das atividades de vida diária, com uma diferença estatisticamente significativa $(p<0,05)$.

\section{f) Cornell-Brown Scale for Quality of Life in Demen- tia (CBS) ${ }^{12}$}

É uma adaptação da Cornell Scale for Depression in Dementia, acrescentando parâmetros que avaliam satisfações, experiências e emoções positivas que ocorreram no último mês, e alterando a variação das pon- tuações que passaram a ser de -2 (pólo negativo extremo) a +2 (pólo positivo extremo).

O paciente e o cuidador são entrevistados em conjunto e as respostas são analisadas por um profissional clínico, com uma pontuação total única. Tem uma duração de cerca de 10-20 minutos. Apresenta 19 questões, estando indicada para pacientes com demência ligeira a moderada. Não inclui perguntas relacionadas com capacidades cognitivas nem com o estado funcional. $\mathrm{O}$ valor total da pontuação varia de $-38 \mathrm{a}+38$. As pontuações negativas indicam que perturbações mentais, comportamentos indesejáveis, problemas de humor e sintomas físicos negativos excedem as avaliações positivas. Logo, a qualidade de vida é pior quanto mais negativa a pontuação.

Os estudos preliminares de fiabilidade e viabilidade foram realizados em 50 doentes com demência e seus cuidadores. A consistência interna (alfa de Cron$b a c h=0,81)$ e a fiabilidade $(r=+0,90)$ foram adequadas, com valores semelhantes entre os pacientes com demência ligeira e demência moderada. Foi encontrada validade de critério pela correlação entre valores da CBS e avaliações visuais de humor dos pacientes $(r=0,63)$ e por correlação negativa com o grau de gravidade da demência medido pela ClinicalDementia Rating Scale $(r=-0,35)$.

\section{g) Dementia Care Mapping (DCM) ${ }^{18}$}

É uma ferramenta observacional desenvolvida para ser usada em pacientes com demência moderada a grave. São avaliadas as manifestações comportamentais do indivíduo por observação intensiva, com registo a cada cinco minutos, por um período de seis horas, através de uma escala de seis pontos.

Os estudos realizados com 177 doentes indicaram haver coerência interna da medida, com correlação significativa entre as pontuações de atividades e estado de saúde.

Os testes de fiabilidade foram realizados com 54 pacientes, durante um período de uma a quatro semanas, com bons resultados para o estado de saúde $(r=0,55)$ e moderados para as atividades $(r=0,40)$.

Foi encontrada uma correlação forte e significativa $(p<0,001)$ entre as pontuações do estado de saúde e a Blau's Generic Measure of QoL $(r=0,73)$. As atividades dos pacientes correlacionaram-se de uma forma fraca com as medidas de QoL $(r=0,29)$. 


\section{h) Psychological Well-Being in Cognitively Impaired} Persons (PWB-CIP) ${ }^{19-20}$

É um questionário de avaliação da QoL mais restrito, que incide apenas sobre o estado emocional e os comportamentos afetivos. Deve ser respondido pelo cuidador, ocupando dez minutos em média. Está indicado para pacientes com demência ligeira a moderada, não institucionalizados. No entanto, como é respondido pelo cuidador, os autores afirmam que também pode ser usado em pacientes com défice cognitivo severo. Inicialmente era composto por 16 questões, que se reduziram a 11 após análise fatorial.

Na primeira análise realizada com 96 pares de participantes (doentes com demência e seus cuidadores), a coerência interna das três áreas de medida (agitação, interações positivas e expressões de descontentamento) foi boa (alfa de Cronbach=0,79; 0,74 e 0,66, respetivamente). Numa segunda avaliação realizada após 18 meses, os resultados obtidos foram semelhantes (alfa de Cronbach $=0,75 ; 0,83$ e 0,68, respetivamente).

Foram encontradas correlações significativas entre as pontuações totais do PWB-CIP e as medidas de depressão $(r=-0,46)$, comportamento social produtivo $(r=0,56)$, estado mental $(r=0,33)$, hostilidade $(r=-0,41) \mathrm{e}$ extroversão $(r=0,56)$.

\section{i) $\mathrm{DEMQOL}^{21}$}

É um questionário criado em Inglaterra, composto por duas partes que se complementam: DEMQoL-28 e DEMQoL-Proxy. A sua construção baseou-se numa revisão da literatura sobre QoL e demência, entrevistas a doentes com demência e a seus cuidadores e opinião de peritos da área. O modelo conceptual inicial incluiu cinco áreas: atividades diárias e de autocuidado, saúde e bem-estar, funcionamento cognitivo, relações sociais e autoconceito. Deve ser considerada a última semana para o seu preenchimento.

No modelo atual, a primeira parte (DEMQoL-28) é preenchida pelo paciente, sendo constituída por 28 questões específicas e uma questão global de QoL. A segunda parte (DEMQoL-Proxy), com 31 questões específicas e uma questão global de QoL, deve ser respondida pelo cuidador. Nos pacientes com demência grave (MMSE < 10) apenas está recomendada a DEMQoL-Proxy. As respostas são classificadas em quatro opções, que variam de nada a muito. Quanto melhor a pontuação, melhor a QoL.

A coerência interna foi elevada para o DEMQoL-28 (alfa de Cronbach $=0,94$ ) e para o DEMQol-Proxy (alfa de Cronbach=0,90).

\section{j) The Quality of Life in Late-Stage Dementia (QUALI- DEM) ${ }^{22}$}

Questionário original na língua holandesa, desenvolvido para os profissionais avaliarem pacientes em diferentes estádios de demência. É composto por 40 perguntas sobre os comportamentos da semana anterior, sendo classificadas numa escala que varia de 0 a 4 , em frequência como nunca, raramente/uma vez por semana, às vezes/poucas vezes por semana, frequentemente/quase diariamente ou diariamente. Do total de questões, 21 foram adaptadas para pacientes com demência avançada.

Foram realizados testes não paramétricos pelo modelo de Mokken, numa amostra de 238 pessoas com demência, com cálculo dos índices de escalonabilidade $(H)$. Os índices de escalonabilidade para as dimensões «sentimentos positivos», «sentimentos negativos», «comportamentos hostis» e «relações sociais» foram fortes $(0,50<H<0,63)$; para «atendimento e cuidados», «autoimagem positiva», «sentir-se em casa» $\mathrm{e}$ «ter alguma coisa para fazer» foram moderados $(0,40<H$ $<0,49)$ e para 0 «isolamento social» foi fraco $(H=0,34)$. $\mathrm{O}$ coeficiente de confiabilidade Rho variou entre 0,60 para o «isolamento social» a 0,90 para «sentimentos positivos» (alfa de Cronbach variando de 0,59 a 0,89).

\section{Questionários de avaliação da QoL na demência validados em Portugal}

\section{a) QOL-AD versão portuguesa ${ }^{8}$}

Em 2012, a autora propôs-se a um processo de tradução e adaptação transcultural da escala QOL-AD, de acordo com as recomendações internacionais. A escala foi aplicada a uma amostra de 104 doentes com demência e seus cuidadores. Foram incluídos doentes com défice cognitivo ligeiro $(n=35)$ e demência ligeira a moderada (MMSE $\geq 10 ; n=69$ ).

Foram estudadas a aceitabilidade, fiabilidade, validade de critério concorrente e discriminativa das escalas.

A versão portuguesa da QOL-AD revelou boa consistência interna ( $\alpha$ Cronbach para as versões do doen- 
te QOL-ADd e do cuidador QOL-Adc de 0,867 e 0,858, respetivamente) e estabilidade teste-reteste com duas semanas de intervalo (CCI: QOL-ADd=0,916, $p<0,001$; $\mathrm{QOLADc}=0,923, p<0,001)$. Foram observadas correlações da QOL-ADd com depressão do doente, satisfação com a vida, funcionalidade e comorbilidades. A QOL-ADc apresentou correlação significativa com sobrecarga do cuidador, sintomas neuropsiquiátricos, depressão do cuidador, estado cognitivo, funcionalidade para atividades instrumentais de vida diária e depressão do doente. Verificou-se a existência de baixa correlação entre a classificação do doente e do cuidador (CCI=0,228 concordância total), apresentando o doente uma classificação de QOL-AD superior à do cuidador. A versão portuguesa da QOL-AD revelou capacidade discriminativa entre uma amostra com e sem défice cognitivo.

Os autores estão convictos que esta escala irá contribuir para o aprofundamento do estudo da QoL dos doentes com demência em Portugal, podendo motivar o desenvolvimento de estratégias para a melhoria da qualidade de vida nestes doentes e suas famílias.

b) DEMQOL (tentativa de validação) ${ }^{23}$

Em junho de 2016 as autoras propuseram-se a um processo de adaptação transcultural do sistema DEMQOL, através de uma revisão da literatura e processo de adaptação dos questionários. O processo implicou a tradução, retroversão, revisão e adaptação cultural à cultura portuguesa.

Os questionários DEMQOL e DEMQOL-Proxy versão portuguesa demostraram boa coerência interna ( $\alpha$ de Cronbach=0,747 e 0,812, respetivamente). Apresentaram excelente reprodutibilidade entre itens para o DEMQOL [ICC $=0,845$; IC95\% $(-0,484-0,984)]$ e para o DEMQOL-Proxy [ICC=0,812; IC95\% (0,636-0,928)]. Foi conseguida a equivalência semântica, conceptual e de conteúdo dos questionários, que mostraram ser indicados para a população portuguesa, com boa coerência interna e reprodutibilidade entre itens.

Devido ao curto período de tempo disponível para a investigação, com $n$ pequeno da amostra face à totalidade dos itens, não foi possível avaliar as validades de constructo e de conteúdo da versão portuguesa dos questionários. Sendo assim, a versão portuguesa do inquérito não ficou validada.

\section{DISCUSSÃO}

Os instrumentos de avaliação da QoL nos indivíduos com demência variam quanto à população a que se aplicam, conteúdo do questionário, quem dá a resposta e tipo de resposta.

A escala mais limitada é a Activity and Affect Indicators of Quality of Life, uma vez que apenas aborda duas dimensões: as atividades realizadas e os estados emocionais.

A maioria dos autores considerou a deterioração cognitiva e a perceção da realidade dos pacientes com demência na decisão da aplicação do questionário. Nos casos ligeiros de demência, o questionário é aplicado aos doentes, podendo também ser aplicado em simultâneo aos cuidadores. Casos de demência grave deverão ser avaliados pelas escalas Activity and Affect Indicators of Quality of Life, QUALID (The Quality of Life in Late-Stage Dementia Scale), DCM (Dementia Care Maping), DEMQOL e QUALIDEM, com informações dadas pelo cuidador. No entanto, ao ser o cuidador a dar a informação, poderá haver uma componente pessoal na sua avaliação e deturpar de forma não intencional a avaliação da QoL do indivíduo em estudo.

Alguns autores aperceberam-se das limitações dos doentes com demência no preenchimento dos questionários. O D-QoL, de Brod, é um questionário breve, simples, com uma escala de resposta visual, para facilidade no preenchimento.

Existem ainda ferramentas de avaliação que permitem uma avaliação transversal ao longo da doença. No entanto, a perceção do conteúdo de uma resposta num estádio inicial da doença poderá não ser idêntica à perceção numa fase mais avançada, enviesando os resultados obtidos.

A maioria dos questionários inclui a dimensão psicológica, comportamental (capacidade cognitiva e funcional), geral e social.

O Quadro I sintetiza as principais características dos questionários validados para avaliação da qualidade de vida na demência.

Existem dois instrumentos com versões validadas em português do Brasil: o QOL-AD e o CBS.

Apenas existe um instrumento de avaliação da QOL na demência com uma versão validada em Portugal o QOL-AD. Ainda existe um longo caminho a percorrer 


\begin{tabular}{|c|c|c|c|c|c|c|c|}
\hline \multirow[t]{2}{*}{ Instrumento de medida } & \multicolumn{2}{|c|}{$\begin{array}{l}\text { Resposta ao } \\
\text { questionário }\end{array}$} & \multicolumn{3}{|c|}{ Grau de demência } & \multirow{2}{*}{$\begin{array}{c}\text { Testes } \\
\text { psicométricos }\end{array}$} & \multirow[t]{2}{*}{ Itens } \\
\hline & Paciente & Cuidador & Ligeira & Moderada & Grave & & \\
\hline DQOL & $x$ & & $x$ & $\mathrm{x}$ & & Sim & 30 \\
\hline Activity \& Affect Indicators & & $x$ & & $x$ & $x$ & Sim & 21 \\
\hline QOL-AD & $\mathrm{x}$ & $x$ & $x$ & $x$ & & Sim & 13 \\
\hline QUALID & & $\mathrm{x}$ & & & $\mathrm{x}$ & Sim & 11 \\
\hline QOLAS & $x$ & & $x$ & $x$ & & Sim & 10 \\
\hline CBS & $\mathrm{x}$ & $\mathrm{x}$ & $x$ & $x$ & & Sim & 19 \\
\hline DCM & & $x$ & & $x$ & $x$ & Sim & - \\
\hline PWB-CIP & & $\mathrm{x}$ & $x$ & $x$ & $\mathrm{Xa}$ & Sim & 11 \\
\hline DEM-QOL & $x$ & $\mathrm{X}$ & $x$ & $x$ & $\mathrm{Xb}$ & Sim & $29 / 32$ \\
\hline QUALIDEM & & $\mathrm{x}$ & $x$ & $x$ & $x$ & Sim & 40 \\
\hline
\end{tabular}

Legenda: $a$ = Como a avaliação é feita pelo cuidador, pode ser aplicado em casos de demência grave; b = Nos casos de demência grave apenas se aplica o questionário ao cuidador.

no sentido de uma amplificação das medidas de avaliação nesta área.

\section{CONCLUSÃO}

A demência é uma doença progressiva, na qual diversos domínios são afetados de forma distinta.

O principal interesse dos instrumentos de avaliação da qualidade de vida na demência deve ser a melhoria do bem-estar destes indivíduos, refletindo todos os domínios afetados pela doença. Deverão incluir áreas como as emoções, autoestima, capacidade funcional, envolvimento com o meio envolvente e relações humanas. Na perspetiva de avaliação dos familiares, as principais dimensões afetadas são a gestão terapêutica, nomeadamente a redução dos efeitos secundários da medicação instituída, como a sedação, e intervenções ligadas à medicina física e reabilitação que promovam autonomia funcional e cognitiva.

O ideal será uma abordagem multidimensional, que comprometa os profissionais para o desenvolvimento de estratégias eficazes que melhorem a QoL das pessoas com demência.

É ainda insuficiente a evidência sobre a correlação entre a QoL e as decisões terapêuticas. São ainda necessários mais estudos para avaliar este impacto, assim como as necessidades por parte dos cuidadores. ${ }^{12}$

É desejável que o avanço da produção científica nesta área tenha em consideração a QoL na avaliação das abordagens terapêuticas.

\section{REFERÊNCIAS BIBLIOGRÁFICAS}

1. Alzheimer Europe. 2013: the prevalence of dementia in Europe [homepage]. Luxembourg: Alzheimer Europe; 2013. Available from: https://www.alzheimer-europe.org/Policy-in-Practice2/Country-comparisons/2013-The-prevalence-of-dementia-in-Europe

2. The WHOQOL Group. The World Health Organization quality of life assessment (WHOQOL): position paper from the World Health Organization. Soc Sci Med. 1995;41(10):1403-9.

3. Carr AJ, Gibson B, Robinson PG. Is quality of life determined by expectations or experience. BMJ. 2001;322:1240-3.

4. Seidl EM, Zannon CM. Qualidade de vida e saúde: aspectos conceituais e metodológicos [Quality of life and health: conceptual and methodological issues]. Cad Saúde Pública. 2004;20(2):580-8. Portuguese

5. Brod M, Stewart AL, Sands L. Conceptualization of quality of life in dementia. In: Albert SM, Longsdon RG, editors. Assessing quality of life in Alzheimer's disease. New York: Springer; 2000. p. 3-16. ISBN 9780826113337

6. Lucas-Carrasco R. Calidad de vida y demencia [Quality of life and dementia]. Med Clin (Barc). 2007;128(2):70-5. Spanish

7. Logsdon RG, Gibbons LE, McCurry SM, Teri L. Assessing quality of life in older adults with cognitive impairment. Psychosom Med. 2002;64(3):510-9.

8. Bárrios HS. Adaptação cultural e linguística e validação do instrumen- 
to QOL-AD para Portugal [dissertation]. Lisboa: Faculdade de Medicina, Universidade de Lisboa; 2012.

9. Lawton MP. Quality of life in Alzheimer disease. Alzheimer Dis Assoc Disord. 1994;8 Suppl 3:138-50.

10. Novelli MM, Nitrini R, Caramelli P.Validation of the Brazilian version of the quality of life scale for patients with Alzheimer's disease and their caregivers (QOL-AD). Aging Ment Health. 2010;14(5):624-31.

11. Logsdon RG, Gibbons LE, McCurry SM, Teri L. Quality of life in Alzheimer's disease: patient and caregivers reports. J Ment Health Aging. 1999;5:21-32.

12. Ready RE, Ott BR, Grace J. Patient versus informant perspectives of quality of life in mild cognitive impairment and Alzheimer's disease. Int J Geriatr Psychiatry. 2004;19(3):256-65.

13. Conde-Sala JL, Garre-Olmo J, Turró-Garrida O, López-Pousa S, VilaltaFranch J. Factors related to perceived quality of life in patients with Alzheimer's disease: the patient's perception compared with that of caregivers. Int J Geriatr Psychiatry. 2009;24(6):585-94.

14. Brod M, Stewart AL, Sands L, Walton P. Conceptualization and measurement of quality of life in dementia: the dementia quality of life instrument (DQoL). Gerontologist. 1999;39(1):25-35.

15. Albert SM, Castillo-Castanada C, Jacobs DM, Sano M, Bell K, Merchant $C$, et al. Proxy-reported quality of life in Alzheimer's patients: comparison of clinical and population- based samples. J Ment Health Aging. 1999;5(1):49-58.

16. Weiner MF, Martin-Cook K, Svetlik DA, Saine K, Foster B, Fontaine CS. The quality of life in late-stage dementia (QUALID) scale. J Am Med Dir Assoc. 2000;1(3):114-6.

17. Selai CE, Trimble MR, Rossor MN, Harvey RJ. Assessing quality of life in dementia: preliminary psychometric testing of the Quality of Life Assessment Schedule (QOLAS). Neuropsychol Rehab. 2001;11(3-4):21943.

18. Fossey J, Lee L, Ballard C. Dementia Care Mapping as a research tool for measuring quality of life in care settings: psychometric properties. Int J Geriatr Psychiatry. 2002;17(11):1064-70.

19. Burgener S, Twigg P. Relationships among caregiver factors and quality of life in care recipients with irreversible dementia. Alzheimer Dis Assoc Disord. 2002;16(2):88-102.

20. Burgener SC, Twigg P, Popovich A. Measuring psychological well-being in cognitively impaired persons. Dementia. 2005;4(4):463-85.

21. Smith SC, Lamping DL, Banerjee S, Harwood R, Foley B, Smith P, et al. Measurement of health-related quality of life for people with dementia: development of a new instrument (DEMQOL) and an evaluation of current methodology. Health Technol Assess. 2005;9(10):1-93.

22. Ettema TP, Dröes RM, de Lange J, Mellenbergh GJ, Ribbe MW. QUALIDEM: development and evaluation of a dementia specific quality of life instrument. Scalability, reliability and internal structure. Int J Geriatr Psychiatry. 2007;22(6):549-56.

23. Jorge $\mathrm{Cl}$, Lopes CD. Qualidade de vida na demência - Adaptação transcultural dos questionários DEMQOL e DEMQOL-Proxy para a população portuguesa: equivalência semântica, conceptual e de conteúdo [monograph]. Leiria: Escola Superior de Saúde, Instituto Politécnico de Leiria; 2016.

\section{CONFLITO DE INTERESSES}

Os autores declaram não ter quaisquer conflitos de interesse.

\section{ENDEREÇO PARA CORRESPONDÊNCIA}

Ana Paula Pinheiro

E-mail: ana_paulapinheiro@hotmail.com

https://orcid.org/0000-0002-8523-7714

Recebido em 17-04-2018

Aceite para publicação em 24-06-2019

\section{ABSTRACT}

\section{INSTRUMENTS FOR ASSESSMENT OF THE QUALITY OF LIFE IN DEMENTIA}

Objectives: This study aimed to acknowledge the specific measures for the assessment of the quality of life (QoL) of patients with dementia. It compares the different measures for various parameters, such as degree of dementia of the target population, psychometric data, and key features.

Data sources: MEDLINE/PubMed and RCAAP reviews.

Methods: Articles published between 1 January 1986 and 31 December 2016 using the MeSH terms Quality of Life, Scales, Instruments, Dementia, and Alzheimer Disease.

Results: Sixty seven papers were obtained, of which 18 were selected. Among these, 10 questionnaires about QoL assessment in individuals with dementia were found.

Conclusions: From the specific instruments found, only one has been translated, adapted and validated for the Portuguese culture.

Keywords: Quality of life; Scales; Measures; Dementia; Alzheimer disease. 